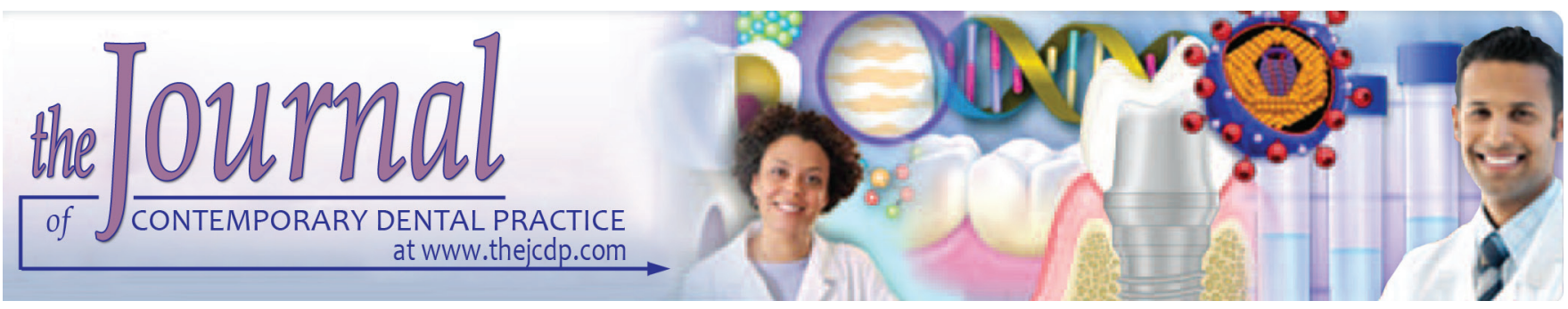

\title{
Immediate and Delayed Effects of Diode Laser on Debonding of Ceramic Brackets: An in vitro Study
}

\author{
${ }^{1}$ Praveena Anand, ${ }^{2}$ PB Anand, ${ }^{3}$ Ramachandran Prabhakar, ${ }^{4} \mathrm{~N}$ Rajvikram, ${ }^{5} \mathrm{P}$ Rajakumar \\ ${ }^{6}$ Varun Reddy Atali, ${ }^{7} \mathrm{R}$ Saravanan
}

\begin{abstract}
Aim: The aim of the study is to evaluate the immediate and delayed effects of diode laser on debonding of ceramic brackets.

Materials and methods: A total of 60 human extracted premolar teeth were randomly assigned to three different treatment groups. All teeth were bonded with adhesive precoated (APC) ceramic brackets (3M Unitek). A total of 20 teeth were debonded without lasing (group 1), 20 immediately after lasing (group 2), and 201 hour after lasing (group 3). For the lasing groups (groups 2 and 3), access cavity was prepared on the occlusal surface to a $2 \mathrm{~mm}$ diameter. A transbond plus self-etching primer (3M Unitek, Monrovia, CA, USA) and APC PLUS clarity advanced brackets (3M, Unitek, Monrovia, CA, USA) were used. The shear bond strength (SBS) and adhesive remnant index (ARI) were measured. The internal pulpal wall temperature was noted for the laser groups.
\end{abstract}

Results: The mean SBS was 15.4, 11.57, and $11.79 \mathrm{MPa}$ for groups 1 to 3 respectively. Post hoc test showed significant difference $(p<0.001)$ between the control group and the lased groups. For groups 2 and 3, the rise in temperature was at an average of 1.4 and $1.3^{\circ} \mathrm{C}$ respectively.

Conclusion: The SBS of APC brackets decreased by $33.3 \%$ on application of diode laser without increasing the internal pulp chamber wall temperature significantly. Shear bond strength remains more or less the same whether debonding is done immediately after lasing or 1 hour after lasing. Diode lasers increased the ARI scores and thus decreased the risk of enamel fracture.

Keywords: Adhesive precoated brackets, Diode laser, Instron machine, Scanning electron microscope, Self-etching primer, Stereomicroscope.

How to cite this article: Anand P, Anand PB, Prabhakar R, Rajvikram N, Rajakumar P, Atali VR, Saravanan R. Immediate and

${ }^{1-7}$ Department of Orthodontics, Thai Moogambigai Dental College and Hospital, Chennai, Tamil Nadu, India

Corresponding Author: P Rajakumar, Reader, Department of Orthodontics, Thai Moogambigai Dental College and Hospital Chennai, Tamil Nadu, India, Phone: +919444466148, e-mail: rajscare@hotmail.com
Delayed Effects of Diode Laser on Debonding of Ceramic Brackets: An in vitro Study. J Contemp Dent Pract 2016;17(4):275-281.

Source of support: Nil

Conflict of interest: None

\section{INTRODUCTION}

With the increase in the number of adults seeking orthodontic treatment and because ceramic brackets are esthetic and cost-effective, the demand for ceramic brackets is increasing. Ceramic brackets are made from alumina (aluminum oxide, $\mathrm{Al}_{2} \mathrm{O}_{3}$ ). Ceramic brackets are manufactured as monocrystalline or polycrystalline. ${ }^{1}$ Both brackets are more esthetic as compared with stainless steel brackets and also resist staining and discoloration. ${ }^{2}$ In addition, ceramic brackets are also available as adhesive precoated (APC) ceramic brackets to reduce the chairside time and the number of bonding steps. ${ }^{3}$

Although ceramic brackets are esthetically superior, their properties, such as decreased tensile resistance, decreased fracture resistance, increased frictional resistance, and increased bond strength ${ }^{4}$ were challenging to orthodontists. Many studies have been carried out since then to rectify their drawbacks and improve their physical properties. ${ }^{5}$

As far as a patient is concerned, the main drawback with ceramic bracket is discomfort and pain at the time of debonding. ${ }^{6}$ Also, there are more chances of enamel cracks. ${ }^{7}$ The force needed to debond brackets is measured as shear bond strength (SBS). Reduced SBS is preferable because greater force creates more discomfort and pain. Several methods have been suggested to debond ceramic brackets, including special pliers, ${ }^{8}$ hand scalers for mechanical debonding, ultrasonic debonding units, ${ }^{9}$ electrothermal debonding devices, ${ }^{10}$ and diamond burs to grind the brackets off the tooth surface. ${ }^{11}$ In the past decade, studies were carried out using lasers (light 
amplification stimulated emission of radiation) for debonding.

Lasers such as $\mathrm{CO}_{2}{ }^{12}$ neodymium-doped yttrium aluminum garnet (Nd-YAG), ${ }^{13}$ and erbium-YAG ${ }^{14}$ were studied for their effects on debonding of ceramic brackets. However, the selection of laser is based on certain factors such as clinical applicability, ease of operation, compactness, and cost-effectiveness. $\mathrm{CO}_{2}$ and Nd-YAG lasers are not used much due to their large size and high cost. Though erbium lasers are smaller in size they are expensive. In clinical practice, a diode laser is preferred due to its soft tissue specificity, simple operation, small size, and relatively low cost. As diode lasers have become a part of clinical setting, the application of laser for debonding ceramic brackets can be studied whether they could be used for easier debonding by softening the adhesive.

\section{AIMS AND OBJECTIVES}

The aims and objectives of the study are (a) to evaluate and compare the SBS and the adhesive remnant index (ARI) of the APC brackets that are debonded without diode lasing, immediately after diode lasing, and 1 hour after diode lasing; (b) to evaluate and compare pulpal wall temperature before and after lasing for the group immediately debonded and the group debonded 1 hour after lasing; and (c) to compare the scanning electron microscope (SEM) images of all the groups.

\section{MATERIALS AND METHODS}

The study used a total of 60 human extracted premolars with intact buccal enamel without any hypoplastic spots, enamel cracks, and caries or restorations. All teeth were rinsed with distilled water and stored in $0.1 \%$ thymol solution. The teeth roots were embedded in acrylic autopolymerizing polymethyl methacrylate (DPI RR cold cure) and acrylic liquid (DPI RR cold cure), leaving the entire crown exposed. The sample size was 20 per group (groups 1 to 3 ) similar to a recently published

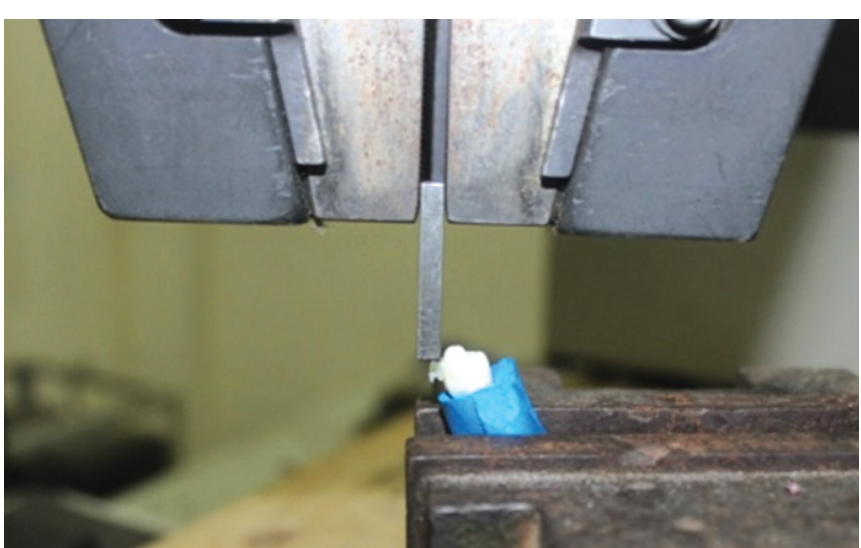

Fig. 1: Orientation of bracket slot in the Instron machine article on diode laser debonding, in which the authors obtained sufficient power to achieve $\mathrm{p}<0.001$ significant difference. ${ }^{15}$ For the lasing groups (groups 2 and 3), access cavity was prepared to a $2 \mathrm{~mm}$ diameter to assess the pulpal wall temperature. After prophylaxis of teeth with an oil-free pumice, the teeth were rinsed with water. Excess water was removed with gentle air burst, but the tooth surface was not overdried.

A transbond plus self-etching primer (3M Unitek, Monrovia, CA, USA) was used. Immediately after etching, APC PLUS clarity advanced brackets (3M, Unitek, Monrovia, CA, USA) were bonded. The brackets were placed onto the tooth surface such that the bracket slots were parallel to the acrylic block and then pressed firmly. The curing light, Ledition (Ivoclar, Vivadent), was held perpendicular to the ceramic surface for 5 seconds. The teeth were stored for 24 hours in distilled water in Isotemp Standard Lab Incubator (CI-10S) at $37^{\circ} \mathrm{C}$.

For group 1, the teeth were debonded without lasing; for group 2, the teeth were debonded immediately after lasing; and for group 3, the teeth were debonded 1 hour after lasing. Upon removal from the $37^{\circ} \mathrm{C}$ incubator, the prepared teeth with the bonded ceramic brackets of the control group were positioned in an SBS Instron Testing Machine Model No-3382. The tooth was oriented in such a way that the bracket slots were parallel to the horizontal axis of the blade at the bracket pad-enamel interface (Fig. 1). Shear bond strength was assessed for all the samples of the control group.

For group 2 samples, the tooth was mounted on the testing machine and the temperature (T1) was assessed by placing the tip of the thermocouple device (R K Temp Sensor, Chennai, India) (Fig. 2) into the prepared access cavity. Then the laser (AMD Lasers, Chennai, India) with the wavelength of $810 \pm 10 \mathrm{~nm}$ at $2.5 \mathrm{~W}$ was pointed at the bracket pad-enamel interface and the pad was lased for 5 seconds (Fig. 3). The maximum pulpal wall temperature during lasing was recorded and the SBS test was performed immediately.

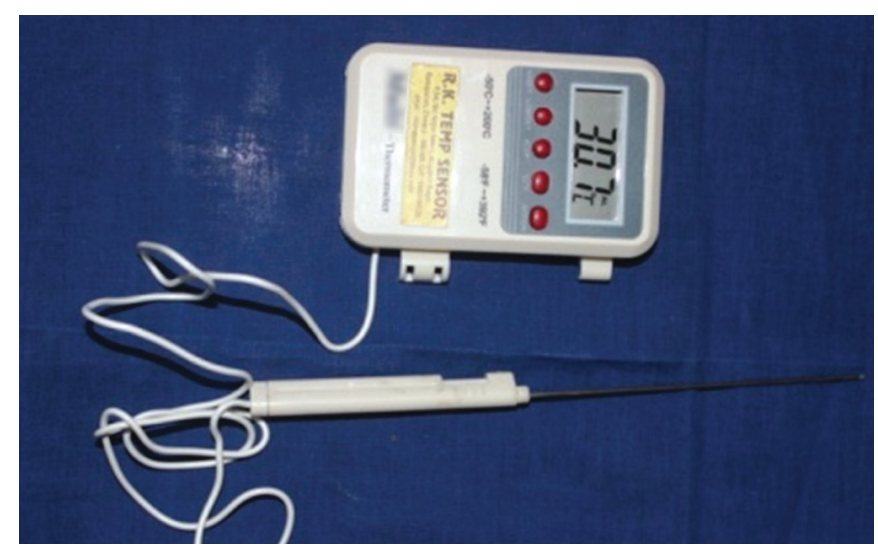

Fig. 2: Thermocouple device 


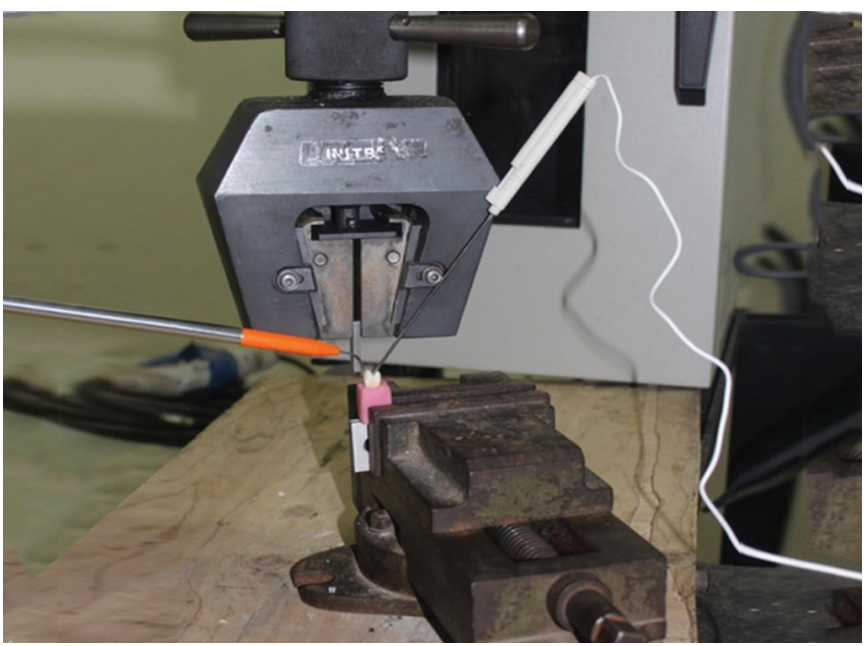

Fig. 3: Assessment of temperature at the time of lasing for group 2

For group 3, the temperature before lasing (T1) was assessed. Then the temperature during lasing (T2) was measured at the time of lasing. Then the samples were incubated for 1 hour. After 1 hour the temperature after lasing (T3) of the samples was measured. Then the SBS test was performed. All the SBS values were tabulated.

The debonded samples were evaluated using a stereomicroscope (zoomstar GO 11017913) at 20× magnification to determine the ARI score. The scale for ARI ranged from 0 to 3: 0 - no adhesive remained on the tooth; 1 - less than $50 \%$ of the enamel bonding surface had composite; 2 - more than $50 \%$ of the enamel bonding surface had composite; 3 - the entire enamel bonding surface had composite.

The samples were prepared for SEM study by sectioning the crown and drying it in the hot air oven at $100^{\circ} \mathrm{C}$ for 1 hour. The specimen was then sputter coated and placed in the SEM (Model No EVO MA 15) and the images were viewed in the SMART software.

\section{RESULTS}

The SBS values in Newtons were converted into MPa by dividing the value by the base surface area of the bracket. The base surface area of APC PLUS clarity advanced bracket was $14.6 \mathrm{~mm}^{2} .^{3}$ The mean SBS was 15.4, 11.57, and $11.79 \mathrm{MPa}$ for groups 1 to 3 respectively. Statistical analysis (analysis of variance, ANOVA) showed that the mean SBS with laser debonding was

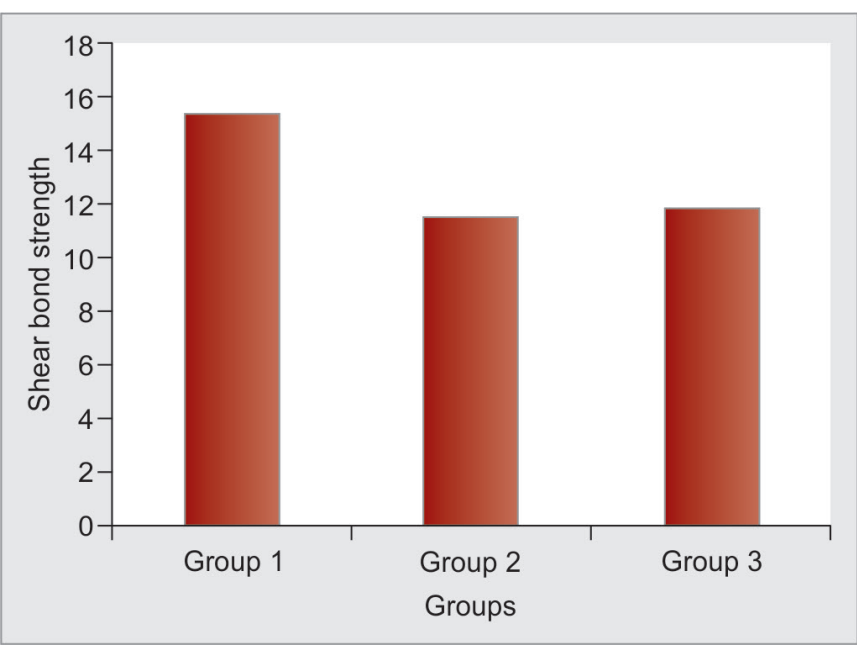

Graph 1: Comparison of mean shear bond strength values. group 1 Debonded without laser; group 2 - Debonded immediately after lasing; group 3 - Debonded 1 hour after lasing

$33.33 \%$ less than that of debonding without lasers $(\mathrm{p}<0.001)$ (Graph 1).

The Tukey Honestly Significant test (post hoc test) (Table 1) was performed for multiple comparisons between the groups. There was significant difference $(\mathrm{p}<0.001)$ between the control group and the lased groups. There was no statistical difference between the immediately debonded lased group and the 1 hour delayed debonded lased group $(\mathrm{p} \leq 0.9)$.

For group 2, the mean temperature values showed that the average rise in temperature was $1.3^{\circ} \mathrm{C}$. Paired t-tests was done, which showed a rise in temperature in group $2(p<0.01)$ (Table 2 and Graph 2).

For group 3, the rise in temperature between $\mathrm{T} 1$ and $\mathrm{T} 2$ was at an average of $1.4^{\circ} \mathrm{C}$ and the temperature rise before (T1) and 1 hour after lasing (T3) was more or less the same. After 1 hour of lasing the mean temperature fall was $1.5^{\circ} \mathrm{C}$. Paired t-tests showed a significant rise

Table 1: Comparison of mean values of shear bond strength

\begin{tabular}{lllll}
\hline (I) Groups & $(J)$ Groups & Mean difference $(I-J)$ & Std. error & Sig. \\
\hline Group 1 & Group 2 & $3.840\left(^{*}\right)$ & 0.7265 & 0.000 \\
& Group 3 & $3.620\left(^{*}\right)$ & 0.7265 & 0.000 \\
Group 2 & Group 1 & $-3.840\left(^{*}\right)$ & 0.7265 & 0.000 \\
& Group 3 & -0.220 & 0.7265 & 0.951 \\
Group 3 & Group 1 & $-3.620\left(^{*}\right)$ & 0.7265 & 0.000 \\
& Group 2 & 0.220 & 0.7265 & 0.951 \\
\hline
\end{tabular}

*The mean difference is significant at the 0.05 level

Table 2: Comparison of mean temperature values of group 2

\begin{tabular}{|c|c|c|c|c|c|c|c|c|}
\hline \multirow[b]{3}{*}{ Pair 1} & \multicolumn{5}{|c|}{ Paired differences } & \multirow[b]{3}{*}{$T$} & \multirow[b]{3}{*}{ Df } & \multirow{3}{*}{$\begin{array}{l}\text { Sig. } \\
\text { (2-tailed) }\end{array}$} \\
\hline & \multirow[b]{2}{*}{ Mean } & \multirow[b]{2}{*}{ Std. deviation } & \multirow[b]{2}{*}{ Std. error mean } & \multicolumn{2}{|c|}{$95 \%$ confidence interval of the difference } & & & \\
\hline & & & & Lower & Upper & & & \\
\hline $\mathrm{T} 1-\mathrm{T} 2$ & -1.325 & 0.5757 & 0.1287 & -1.594 & -1.056 & -10.293 & 19 & 0.000 \\
\hline
\end{tabular}




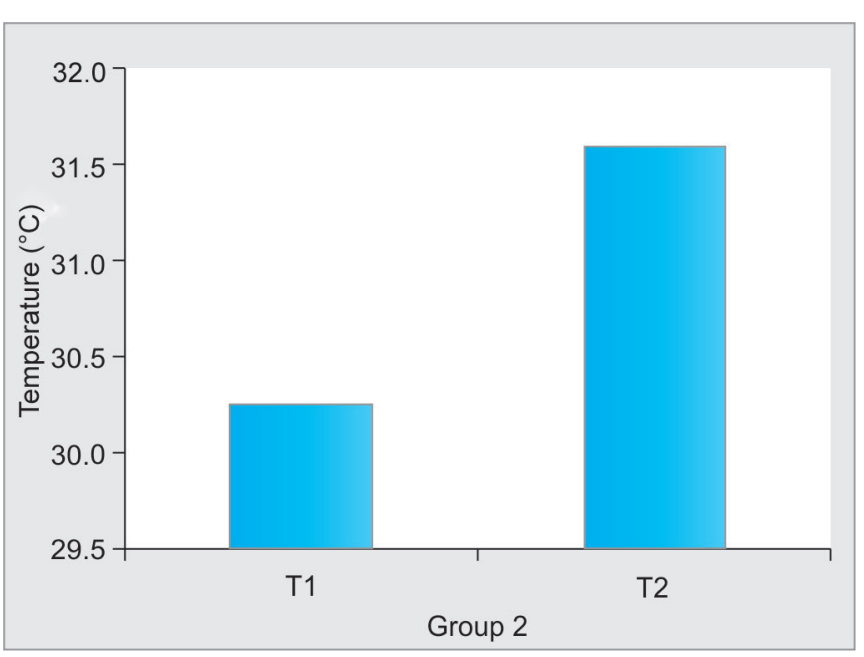

Graph 2: Comparison of mean temperature of group 2. T1 Temperature before lasing of group 2; T2 - Temperature at the time of lasing and debonding of group 2

between the temperature before lasing and at the time of lasing $(\mathrm{p}<0.01)$. There was no significant difference between the temperature before lasing and 1 hour after lasing $(\mathrm{p}=0.908)$. There was a significant difference (fall in temperature) between the temperature at the time of lasing and 1 hour after lasing (Table 3 and Graph 3).

A comparison of mean ARI scores (Fig. 4) shows that groups 2 and 3 received more ARI score than group 1 (ANOVA, $\mathrm{p}=0.18$ ). The Tukey Honestly Significant test

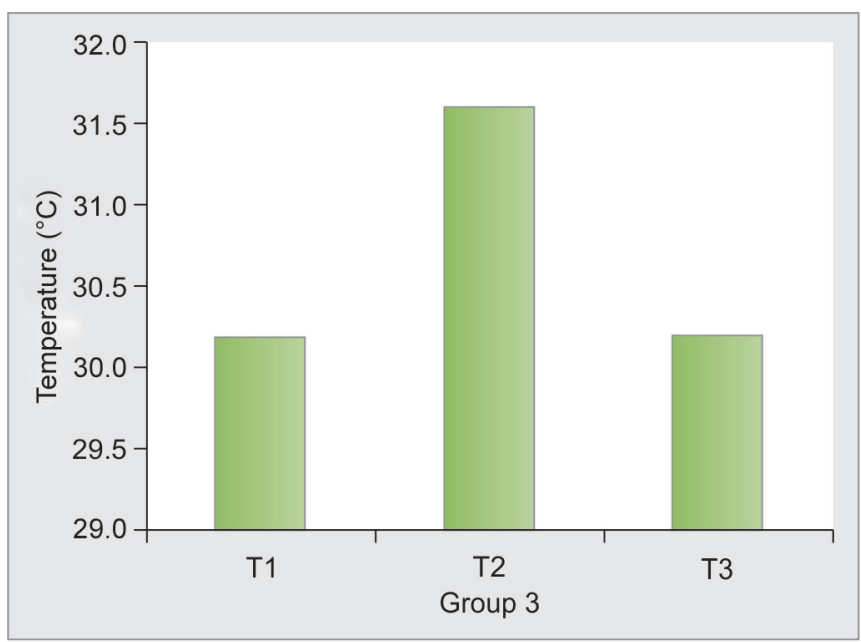

Graph 3: Comparison of temperature values of group 3. T1 Temperature before lasing; $\mathrm{T} 2$ - Temperature at the time of lasing; T3 - Temperature at the time of debonding

was performed for multiple comparisons between the groups. There was less significant difference between groups 2 and $3(p<1)$. On comparing each score between the groups, group 1 received more of score 1 , group 2 had more of score 2, and group 3 had more of score 3.

The SEM image reveals that the possibility for the enamel cracks to occur was more with the control group (samples not lased) as compared with the other groups (Fig. 5).

Table 3: Comparison of temperature values of group 3

\begin{tabular}{|c|c|c|c|c|c|c|c|c|c|}
\hline & & & & Paired diffe & ces & & & & \\
\hline & & & & & $\begin{array}{r}95 \% \text { cc } \\
t\end{array}$ & $\begin{array}{l}\text { terval of } \\
\text { ce }\end{array}$ & & & \\
\hline & & Mean & $\begin{array}{l}\text { Std. } \\
\text { deviation }\end{array}$ & $\begin{array}{l}\text { Std. error } \\
\text { mean }\end{array}$ & Lower & Upper & $T$ & $D f$ & $\begin{array}{l}\text { Sig. } \\
\text { (2-tailed) }\end{array}$ \\
\hline Pair 1 & $\mathrm{~T} 1-\mathrm{T} 2$ & -1.425 & 0.6897 & 0.1542 & -1.748 & -1.102 & -9.240 & 19 & 0.000 \\
\hline Pair 2 & $\mathrm{~T} 1-\mathrm{T} 3$ & -0.010 & 0.3824 & 0.0855 & -0.189 & 0.169 & -0.117 & 19 & 0.908 \\
\hline Pair 3 & $\mathrm{~T} 2-\mathrm{T} 3$ & 1.415 & 0.8381 & 0.1874 & 1.023 & 1.807 & 7.551 & 19 & 0.000 \\
\hline
\end{tabular}

T1 - Temperature before lasing; T2 - Temperature at the time of lasing; T3 - Temperature at the time of debonding
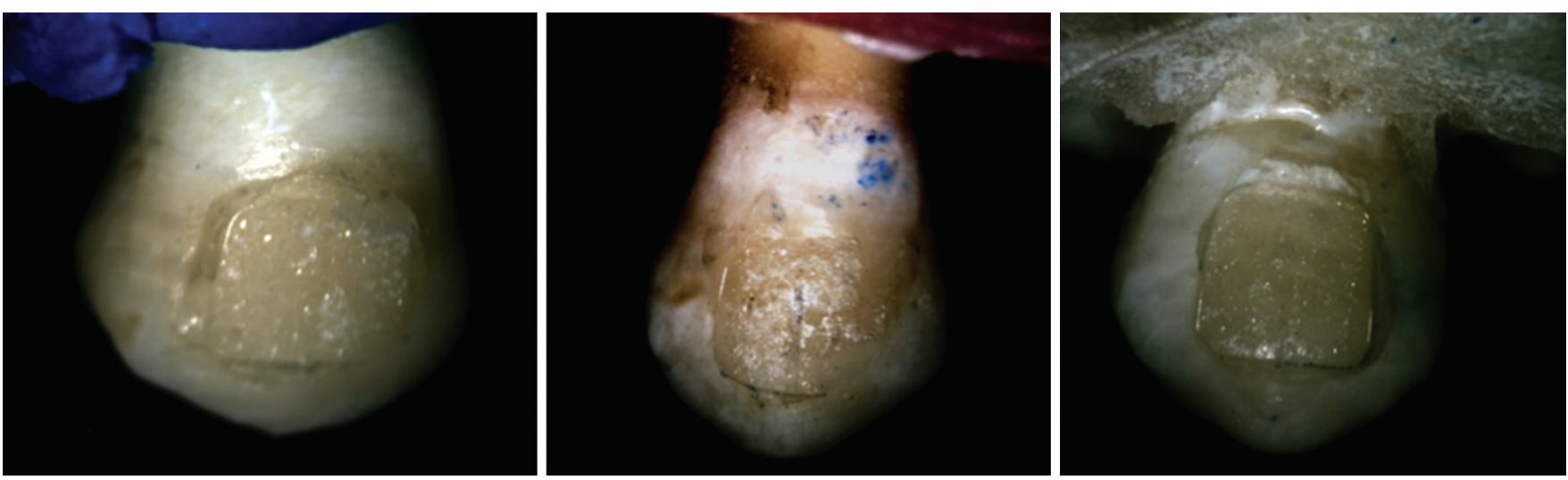

Fig. 4: Stereomicroscope images of groups 1 to 3 samples 

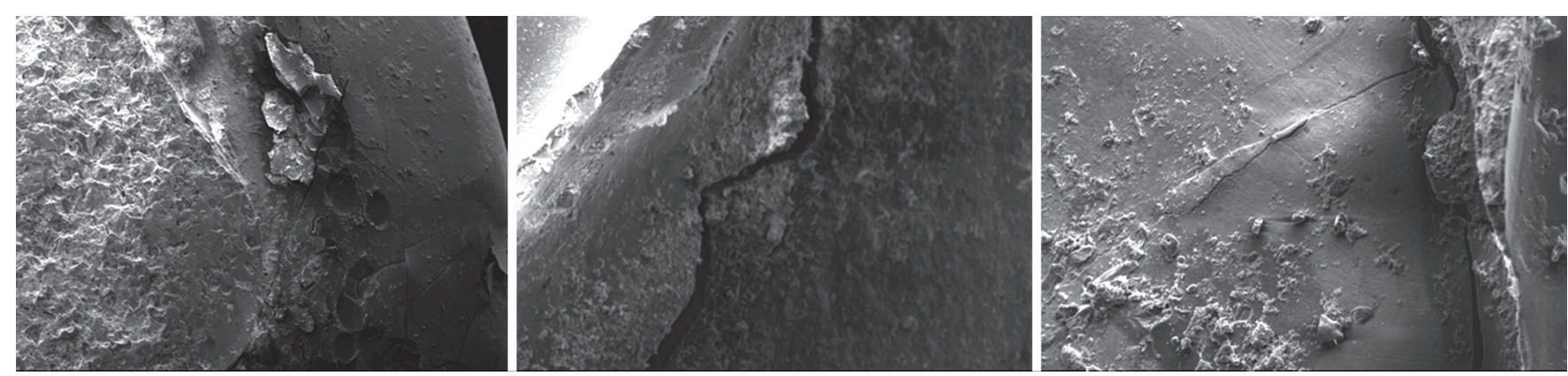

Fig. 5: Scanning electron microscope image of group 1 (under $40 x$ ) and groups 2 and 3 (under 200x)

\section{DISCUSSION}

With the advent of ceramic brackets in the 1980s, many studies were carried out to assess its physical and mechanical properties. The main drawback with ceramic brackets is the patient's discomfort at the time of debonding. ${ }^{6}$ The debonding of ceramic brackets can increase the risk of enamel damage. ${ }^{7}$

The combination of the primer/adhesive and bracket pad design must not produce SBS that is too low to prevent the bracket from falling off the teeth prematurely or too high that they can fracture the enamel during debonding. Shear bond strength should be within the range of 5.9 to $7.8 \mathrm{MPa}$ as being studied by Reynolds and von Fraunhofer, ${ }^{16}$ an effective way to debond ceramic brackets.

The present study of debonding ceramic brackets is carried out based on the fact that the lasers can be used for debonding. ${ }^{12}$ Among different lasers, diode lasers are excellent soft tissue surgical lasers and indicated for incising, excising, and coagulating gingiva and mucosa. ${ }^{17}$ Also diode lasers are simple to use, cost-effective, and compact. As diode lasers are used in everyday practice, their application on debonding ceramic bracket will be an added advantage to the practitioner. Hence, the study was planned to evaluate the effect of diode laser on the debonding strength of ceramic brackets.

To reduce the chairside time, self-etching primer (SEP) was used on extracted human premolar teeth. According to Banks and Thiruvenkatachari, ${ }^{18}$ there was no difference in the failure rates of brackets bonded with SEP or conventional etch and primer; also bonding with SEP was significantly faster than using conventional etch and primer. Hence, in the current study, SEP (Transbond Plus, 3M Unitek) was used.

To improve work efficiency and to decrease the chairtime, the manufacturers have come up with a new generation of APC ceramic brackets. Precoated brackets provide a more uniform adhesive thickness and reduce the number of bonding steps. ${ }^{3}$ As the standardization of the thickness of the composite material is an important factor to study SBS, APC ceramic brackets (APC PLUS clarity advanced brackets, 3M Unitek) (polycrystalline brackets) were used.
Though APC clarity advanced bracket has a stressconcentrator vertically on the bracket base to facilitate easy debonding, further decrease in bond strength before debonding could help in the reduction of enamel cracks.

The shear test revealed that SBS had lowered significantly in the lased group. This may be explained by the fact that a diode laser is a semiconductor device that produces coherent radiation (in which the waves are all at the same frequency and phase) when current passes through it. Laser-initiated debonding works by degrading or thermally softening the adhesive resin. ${ }^{19}$ This finding is in agreement with other studies that concluded that the diode laser debonding protocol used did not produce any explosive "blow-offs," unlike the changes produced by Nd-YAG laser such as carbonization to the remnant resin or decomposition of the bracket base. ${ }^{13}$ The results of this study agree with those of previous studies, substantiating the fact that lasers can be used effectively to thermally soften the adhesive resin for the removal of ceramic brackets. ${ }^{20}$

The present study is contradictory to the study carried out by Feldon et al, ${ }^{21}$ in which diode laser used at 2 and $5 \mathrm{~W}$ significantly decreased the debonding force for monocrystalline brackets without increasing the pulp chamber temperature significantly and there was no significant decrease in the debonding force required for polycrystalline brackets, but agrees with the study by Almohaimeed and El Halim, ${ }^{15}$ where the application of diode laser was effective in debonding precoated ceramic brackets in which shear test was performed 24 hours after lasing.

However, there is not much difference between the groups that have been debonded immediately after lasing and 1 hour after lasing. This can be attributed to the fact that the adhesive resin that has been softened by the laser remains soft even after 1 hour of lasing. This shows that debonding can be carried out even 1 hour after lasing.

In the immediately debonded group, the average rise in temperature was $1.3^{\circ} \mathrm{C}$. This shows that the rise in pulpal wall temperature is within the range as the study of Rhesus monkey teeth by Zach and Cohen ${ }^{22}$ showed that when external heat was applied to the teeth 
there was no pulpal damage in the teeth that had an intrapulpal temperature increase of $2.2^{\circ} \mathrm{C}$. When the external heat was increased to cause an intrapulpal temperature increase of $5.5^{\circ} \mathrm{C}$, pulpal necrosis was observed in $15 \%$ of the teeth. When the external heat was increased to cause an intrapulpal temperature increase of $11^{\circ} \mathrm{C}$, pulpal necrosis was observed in $60 \%$ of the teeth. When the intrapulpal temperature was increased to $17^{\circ} \mathrm{C}, 100 \%$ of the teeth developed pulpal necrosis. These observations demonstrate that increased pulpal temperatures can injure the dental pulp.

In the delayed debonded group, the mean rise in temperature before lasing and at the time of lasing was $1.4^{\circ} \mathrm{C}$. However, when debonding 1 hour after lasing, there was a very minimal rise in temperature (mean $0.01^{\circ} \mathrm{C}$ ) as compared with the temperature before lasing. But the SBS was decreased as compared with the control group. This can be attributed to the fact that the adhesive remains soft at 1 hour as the samples were incubated at $37^{\circ} \mathrm{C}$.

According to Odegaard and Segner, ${ }^{23}$ the ceramic brackets showed superior SBS when compared with the metal brackets. Moreover, the failure site for the metal brackets mainly occurred in the bracket-adhesive interface, while the bond failure with the ceramic brackets occurred primarily in the enamel-adhesive interface. In another study by Hajrassie and Khier, ${ }^{24}$ the ARI analysis revealed that $60 \%$ of ceramic bracket samples had no adhesive left on the tooth. As a result, higher direct impact force on the enamel surface during debonding could be expected, which may increase the risk of causing enamel damage and/or fracture.

In the current study, the mean ARI score showed that the lased group received higher score as compared with the control group. Hence, the chance for enamel cracks to occur is more with control group than other groups. Additionally, the ARI scores were almost within the secure range, similar to previous studies. ${ }^{12,19}$

The SEM image revealed that the possibility for enamel cracks to occur was more with the control group (samples not lased) as compared with the other groups. This goes with the study carried out by Almohaimeed and El Halim, ${ }^{15}$ in which enamel cracks were less for the samples that had been lased.

The limitation of this study could be the duration of laser application. The average SBS obtained in the study was $11.57 \mathrm{MPa}$ as compared with the normal SBS ranging between 5.9 and $7.8 \mathrm{MPa},{ }^{16}$ and the average rise in temperature $\left(1.5^{\circ} \mathrm{C}\right)$ was within the limit as by the Zach and Cohen study. ${ }^{22}$ So there is the possibility of reducing SBS further if the duration of lasing is increased or using a high-power diode laser. As a result there may be chances for ARI scores to reduce without enamel damage. Then the time taken for cleaning procedures will also be reduced.

Further study is needed on bracket design, duration of diode lasing, and level of diode laser so that debonding can be carried out more effectively with minimal patient's discomfort.

\section{CONCLUSION}

The debonding of ceramic brackets is often associated with the patient's discomfort and pain. Application of lasers in debonding of ceramic brackets is an effective innovation. Diode lasers being portable, simple to operate, and low cost, and because of their wide use as soft tissue laser in clinical practice, their application in debonding is very useful. From this study, it can be concluded that

- The SBS of APC brackets decrease by $33.3 \%$ on application of diode laser without increasing the internal pulp chamber wall temperature significantly.

- Shear bond strength remains more or less the same whether debonding is done immediately after lasing or 1 hour after lasing.

- Diode lasers increased the ARI scores and thus decreased the risk of enamel fracture.

The limitation in this study could be the duration of laser application, which can be increased to reduce the ARI scores, so that the time taken for cleaning procedures will be reduced. Further study is needed on bracket design, duration of diode lasing, and level of diode laser so that debonding can be carried out more effectively with minimal patient's discomfort. These may improve the practice of orthodontics.

\section{REFERENCES}

1. Swartz ML. Ceramic brackets. J Clin Orthod 1998 Feb;22(2): 82-88.

2. Russell JS. Aesthetic orthodontic brackets. J Orthod 2005 Jun;32(2):146-163.

3. Bishara SE, Oonsombat C, Soliman MM, Warren JJ, Laffoon JF, Ajlouni R. Comparison of bonding time and shear bond strength between a conventional and a new integrated bonding system. Angle Orthod 2005 Mar;75(2):237-242.

4. Kusy RP. Morphology of polycrystalline alumina brackets and its relationship to fracture toughness and strength. Angle Orthod 1988 Jul;58(3):197-203.

5. Rhodes RK, Duncanson MG Jr, Nanda RS, Currier GF. Fracture strength of ceramic brackets subjected to mesialdistal arch wire tipping forces. Angle Orthod 1992 Spring; 62(1):67-76.

6. Williams OL, Bishara SE. Patient discomfort levels at the time of debonding: a pilot study. Am J Orthod Dentofacial Orthop 1992 Apr;101(4):313-317.

7. Pont HB, Özcan M, Bagis B, Ren M. Loss of surface enamel after bracket debonding: an in vivo and ex-vivo evaluation. Am J Orthod Dentofacial Orthop 2010 Oct;138(4): 387e1-387e9. 
8. Bishara SE, Fehr DE. Comparisons of the effectiveness of pliers with narrow and wide blades in debonding ceramic brackets. Am J Orthod Dentofac Orthop 1993 Mar;103(3): 253-257.

9. Bishara SE, Trulove TS. Comparisons of different debonding techniques for ceramic brackets: an in vitro study Part 1. Background and methods. Am J Orthod Dentofacial Orthop 1990 Aug;98(2):145-153.

10. Cummings M, Biagioni P, Lamey P-J, Burden DJ. Thermal image analysis of electrothermal debonding of ceramic brackets: an in vitro study. Eur J Orthod 1999 Apr;21(2): 111-118.

11. Vukovich ME, Wood DP, Daley TD. Heat generated by grinding during removal of ceramic brackets. Am J Orthod Dentofacial Orthop 1991 Jun;99(6):505-512.

12. Strobl K, Bahns TL, Willham L, Bishara SE, Stwalley WC. Laser-aided debonding of orthodontic ceramic brackets. Am J Orthod Dentofacial Orthop 1992 Feb;101(2):152-158.

13. Hayakawa K. Nd:YAG laser for debonding ceramic orthodontic brackets. Am J Orthod Dentofacial Orthop 2005 Nov;128(5): 638-647.

14. Oztoprak MO, Nalbantgil D, Erdem AS, Tozlu M, Arun T. Debonding of ceramic brackets by a new scanning laser method. Am J Orthod Dentofacial Orthop 2010 Aug;138(2): 195-200.

15. Almohaimeed M, El Halim SA. Diode laser de-bonding of pre-coated ceramic brackets. J Am Sci 2013;9(5s):177-181.
16. Reynolds IR, von Fraunhofer JA. Direct bonding of orthodontic attachments to teeth: the relation of adhesive bond strength to mesh size. Br J Orthod 1976 Apr;3(2):91-95.

17. Sarver DM. Principles of cosmetic dentistry in orthodontics: part 1. Shape and proportionality of anterior teeth. Am J Orthod Dentofacial Orthop 2004 Dec;126(6):749-753.

18. Banks $P$, Thiruvenkatachari B. Long term clinical evaluation of bracket failure with a self-etching primer: a randomized clinical trial. J Orthod 2007 Dec;34(4):243-251.

19. Mimura H, Deguchi T, Obata A, Yamagishi T, Ito M. Comparison of different bonding materials for laser debonding. Am J Orthod Dentofacial Orthop 1995 Sep;108(3):267-273.

20. Rickabaugh JL, Marangoni RD, McCaffrey KK. Ceramic bracket debonding with the carbon dioxide laser. Am J Orthod Dentofacial Orthop 1996 Oct;110(4):388-393.

21. Feldon PJ, Murray PE, Burch JG, Meister M, Freedman MA. Diode laser debonding of ceramic brackets. Am J Orthod Dentofacial Orthop 2010 Oct;138(4):458-462.

22. Zach L, Cohen G. Pulp response to externally applied heat. Oral Surg Oral Med Oral Pathol 1965 Apr;19:515-530.

23. Odegaard J, Segner D. Shear bond strength of metal brackets compared with a new ceramic bracket. Am J Orthod Dentofacial Orthop 1988 Sep;94(3):201-206.

24. Hajrassie MK, Khier SE. In vivo and in vitro comparison of bond strengths of orthodontic brackets bonded to enamel and debonded at various times. Am J Orthod Dentofacial Orthop 2007 Mar;131(3):384-390. 